\title{
Researches on the Observation on the Jetting of Liquid of High Viscosity and Droplet Control
}

\author{
Hong-wu Zhan", Fang Xu and Ling-feng Lu
}

Zhejiang University of Technology, Hangzhou, China

\begin{abstract}
Valve regulated droplet jetting is an important method for the generation of liquid droplet of high viscosity. Based on learning from piezoelectric micro-droplet jetting mechanism and researching methods, theoretical analysis and simulation were conducted on the factors affecting the micro-droplet jetting volume, and the qualitative relationship between the size of the jetted droplet and the feed pressure and striker stroke so as to guide the designing of the structure of the pneumatic valve control type nozzle and design the droplet jetting system and observation experiment platform based on stroboscopic techniques. Different jetting parameters was changed according to the results of the theoretical analysis for the conduction of jetting experiment, and several glycerin solutions at different solutions were taken as the working liquid for studying the effect of the several factors like feed pressure, pulse driving pressure, driving pulse width, and striker stroke. The striker would not be lifted to an ultimate position through controlling the pulse air pressure or driving pulse width so as to be able to jet extremely tiny droplet, but this is of rather bad stability. It is the most effective, stable and optimized method to control the striker stroke so as to change the diameter and shapes of the droplets.
\end{abstract}

Keywords: 3D printing, droplet jetting, droplet observation, high viscosity.

The prototype of droplet jet technology could be traced back to the conception to split liquid into droplets proposed by Rayleigh in 1878 [1-3]. After the UK "The Economist" focused on the rapid prototyping and direct manufacturing technology in 2012, the concept of 3 Dimension Printing had been extended to generally refer to various kinds of additive manufacturing processes based on laser and droplet jetting methods, among which the 3 Dimension Printing based on droplet jetting was to jet out the photocuring or hot melting moulding materials in the form of micro-droplets for direct stacking and molding or jet the binder selectively to the thin layer of powder for molding.

The diversity of the structure and the complex of the characters of the working liquid has proposed new requirements on the researches of the jetting structure and mechanism of the micro droplet, and how to enable more kinds of new-typed materials of various kinds of features to be jet out in the form of droplet and in a high effective way and maintain the precise controllability on the shape and volume of the droplet is the key technical issue for the 3D printing property and applied extension.

The working frequency using the audio frequency vibration of the piezoelectric ceramic materials could reach doubles of $\mathrm{KHz}$, which has shown tremendous value for engineering application and scientific researching in traditional ink-jet printing and many special printing fields. However, the commonly used piezoelectric actuator has strictly limited the structural parameters of the working liquid (ink or

*Address correspondence to this author at the Zhejiang University of Technology, Mechanical building B702, No.18, Chaowang Road, Hangzhou, P.R. China; Tel: +86-571-88320957; Fax: +86-571-88320957;

E-mail:waltzhan@hotmail.com printing ink) and it is more appropriate for the jetting of droplets of low viscosity within the scope of several and dozens of picoliters, but it is hard for it to meet the needs in the spraying of more and more complicated and new-typed moulding materials of high viscosity. When fluid of rather high viscosity is jetted at the rate above $200 \mathrm{mPa} \cdot \mathrm{s}$, then pneumatic valve controlled jetting structure is still the most commonly seen scheme. Pneumatic valve controlled jetting is not sensitive to the structure of the working fluid, and it could work rather well within a very large viscosity dynamic scope. However, due to the limitations by working theories, mechanical manufacturing process and material features, this droplet able to be jetted by this structure has a rather large diameter. There were few previous researches on the controlling of the volume and shapes of the droplets before, and this research has conducted theoretical analysis and stimulation on the factors affecting the jetting volume of the droplets based on the learning of the mechanisms and researching of the piezoelectric micro-droplet, specified the qualitative relationship between the size of the jetted droplets and the feed pressure and the striker stroke, guided the designing of the structure of the pneumatic valve controlled nozzle, designed the droplet jetting system, and observed the process of the formation of the micro-droplets on the experimental platform. In addition, it changed different jetting parameters according to the results of theoretical analysis for conducting jetting experiments and studying the effect of several factors, like feed pressure, pulse driving pressure, driving pulse width, and striker strokes, on droplet jetting.

\section{METHOD OF DROPLET JETTING}

\subsection{Conditions of the Jetting of Stimulated Droplets}

In early studies of $3 \mathrm{D}$ printing the continuous jet printing (CIJ) technology was seen more frequently, but CIJ had 


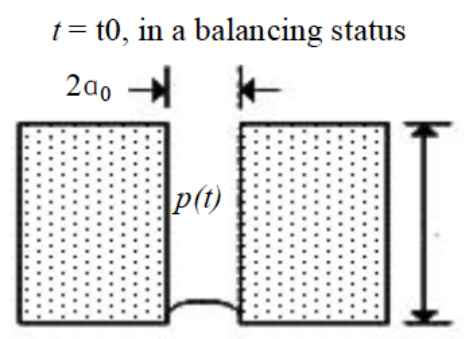

$t=t^{*}$, the pressure within the cavity is at the lartest value

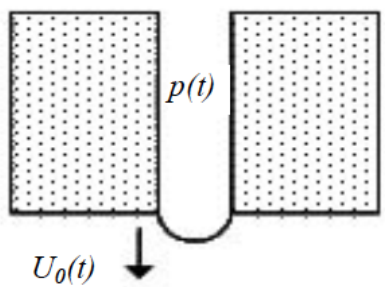

$t>t^{*}$, the droplet is jetted out

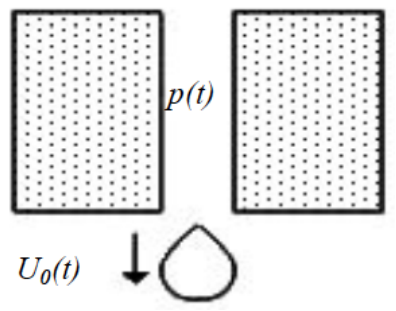

Fig. (1). Simplified mechanical extrusion jetting model.

rather strict requirements on the conductivity, viscosity (4-7 $\mathrm{mPa}$ ) and surface tension $(25-70 \mathrm{mN} / \mathrm{m})$ of the working liquid, thus resulting in rather difficult configuration of the liquid [4]; and the conducting salt added into the working liquid had also reduced the density of the moulding material. Currently the drop on demand (DOD) generating method is usually used, especially the piezoelectric drop on demand. It has been shown by some researches that the liquid was squeezed by the mechanical deformation based on the cavity so as to generate spraying, which was less sensitive to the parameters of the working liquid like temperature and viscosity compared to the method of thermal foaming, thus it could have a broader basis for the adaptability of the liquid and a promising driving methods for the realization of complicated liquid droplet jetting technology.

Fig. (1). is an illustration of the working process of the nozzle jetting droplets in a simplified vacuum [5-7], and the working liquid in the cavity has been stimulated, so the liquid is jetted out from the orifice with the wall height as $L_{0}$.

The process of the formation of the droplets within the model needs to meet two requirements [8-9]:

1) It needs to overcome the surface tension, that is:

$$
\frac{1}{2} \rho U_{0}^{2}\left(t^{*}\right)>2 \sigma / a_{0}
$$

As to the phenomena of satellite droplet, the volume of the jetted droplet is not smaller than that of a droplet, that is:

$$
V_{\text {eject }}=\int_{0}^{t^{*}} U_{0}(t) \pi \alpha_{0}^{2} d t \geq \frac{4}{3} \pi \alpha_{0}^{3}
$$

It is approximately regarded as that the liquid within the cavity and the liquid within the nozzle flows at the same speed, that is, the pressure $p$ within the cavity only has effect on the liquid at the nozzle, and then it could be followed by:

$W_{e} \geq \frac{8}{3} \frac{p a_{0}^{2}}{\sigma L_{0}}$

Among which, $U_{0}(t)$ is the axial velocity of the liquid, $a_{0}$ is the radius of the nozzle, the pressure within the cavity reaches the maximum value at the time point of $a_{0}$, the liquid surface at the nozzle was in the shape of a hemisphere, the Weber number $W_{e}$ means the ratio of the fluid inertia to the surface tension, $p$ is the pressure within the cavity, and $\sigma$ is the liquid surface tension coefficient. The aforementioned conditions must be met to form liquid jetting.

\subsection{Designing of the Pneumatic Valve Controlled Nozzle}

The pneumatic valve controlled nozzle is formed by the springs, seat, piston, strikers, base, and material cylinders, as shown by Fig. (2). When the pneumatic valve controlled nozzle begins to jet, the compressed air A will enter the cavity of the seat to push the piston to go upward, and the spring is gradually compressed. The piston and the striker are as a whole, and in the meantime when the spring is compressed, the striker gradually leaves the position of the bevel within the base and the liquid materials within the material cylinder are automatically complemented to the bevel area of the base under the effect of the compressed air B so as to finish the process of the filling of materials.

The compressed air $\mathrm{A}$ is the pulse pressure and it will disappear after having effect for a while, and at this time the piston will accelerate the downward activity under the elasticity of the spring. Before the striker contacts the base cone, a certain amount of liquid is stuck at the position of the bevel of the base and flow downward through the nozzle due to the extremely large partial pressure. When the striker is knocking the bevel, the liquid is cut and then flow from the nozzle due to the effect of the inertia force.

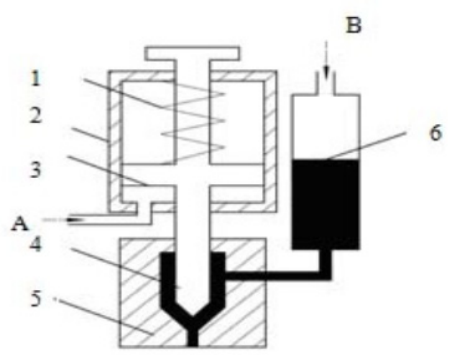

A- Compressed air
B- Compressed air
1- spring
2- seat
3- piston
4- striker
5- base
6- material cylinder

Fig. (2). The structure of the nozzle.

\subsection{Analysis on the Jetting Process}

The liquid within the nozzle is taken as the target for the research, and during the process of the knocking of the striker, the liquid will be affected by the joint influences of feed pressure F1, the impact force $F 2$ of the striker, and the viscous force. $\mathrm{F}$ is the resultant force of $\mathrm{F} 1$ and $F 2$, and the force diagram is shown by Fig. (3).

It could be obtained as below through Newton's Second Law of Motion

$F-F_{\mu}=m a=\rho s l \frac{d v}{d t}$ 
The viscosity of the liquid is

$F_{\mu}=2 \pi r l \tau$

The dynamic viscosity of the liquid is

$\tau=\frac{v \mu}{r}+\tau_{0}$

Then the differential equation of motion of the fluid jetted liquid is :

$p s l \frac{d v}{d t}+2 \pi \mu l v=F-2 \pi r l \tau_{0}$

$v(0)=0$ is taken as the boundary condition, then the fluid kinematic velocity $\mathrm{v}(\mathrm{t})$ is:

$$
\begin{aligned}
& v(t)=\frac{1}{2} \frac{2 \pi r l \tau_{0}-F}{\pi \mu l} e^{-\frac{2 \pi \mu}{p s} t}+\frac{1}{2} \frac{F-2 \pi r l \tau_{0}}{\pi \mu l} \\
& =F-\frac{1}{2} \frac{F-2 \pi r l \tau_{0}}{\pi \mu l}\left(1-e^{-\frac{2 \pi \mu}{p s} t}\right)
\end{aligned}
$$

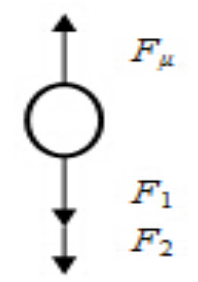

Fig. (3). The droplet mechanical model.

Among which, $\rho$ is the fluid density within the nozzle, $\tau$ is the dynamic viscosity, $\mathrm{s}$ is the nozzle hole area, $\mathrm{r}$ is the radius of the nozzle, and 1 is the length of the nozzle. It could be seen from the aforementioned function that the kinematic velocity of the jetted liquid is in direct proportion to the resultant force and in inversely proportional to the liquid viscosity. Simulation analysis were further conducted and the relationships between the liquid droplet flow velocity in the nozzle and the effort and the intrinsic characteristics as well as the viscosity of the liquid are shown in Fig. (4).

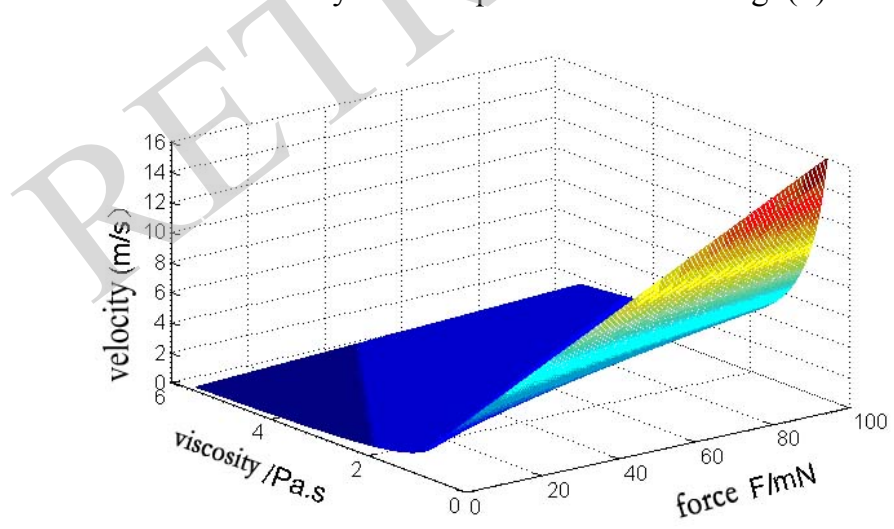

Fig. (4). The relationship among the flow velocity- acting forceviscosity within the nozzle.

The speed of the liquid within the nozzle increases with the increasing of the acting force and decreases with the increasing of the viscosity. As to the liquid at the nozzle, the larger the speed is, the larger the volume of the liquid jetted out within the same time will be. Therefore, the amount of the jetted droplet may be changed through changing the acting force. During the process of the simulation, the radius of the nozzle is set as $r=0.2 \mathrm{~mm}$, the length of the nozzle is set as $l=3 \mathrm{~mm}$, the density of the liquid is set as $\rho=1.59 \mathrm{~g} / \mathrm{cm} 3$, the yield stress is set as $\tau_{0}=200 \mathrm{~Pa}$.

The acting force $\mathrm{F}$ maybe changed through changing the feed pressure $\mathrm{F} 1$ and the compact of the striker $F_{2}$. The spring and the striker are taken as the target for the researching and the following function may be obtained according to the conservation of energy and theorem of momentum:

$$
F_{2}=\frac{\sqrt{m k x^{2}+2 m\left(k x_{0}-f\right) x}}{\Delta t}
$$

It could be seen from Equation (9) that the impact force $F_{2}$ is of non-linear relationship to the stroke $\mathrm{x}$, and the larger the striker stroke is, the stronger the impact force generated would be, just as shown by Fig. (5).

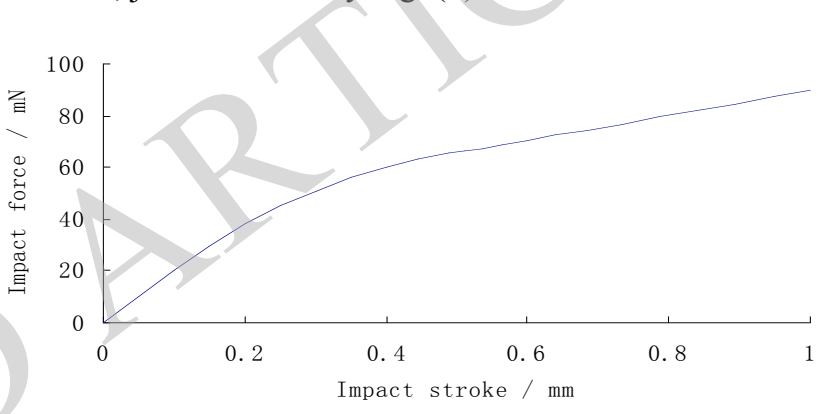

Fig. (5). The relationship between the impact force and the impact stroke.

It could be known through combining the results of the analyses on the stress on the liquid within the nozzle as well as the mechanical analysis of the spring and the striker that the amount of the jetted droplet increases with the increasing of the feed pressure and striker stroke.

\section{EXPERIMENT OF THE OBSERVATION ON THE JETTING OF THE DROPLET}

\subsection{Jetting Experimental Platform}

The experimental platform consists of the driving pulse generation controller, the barometric control system, the temperature controlling system, the pneumatic valve controlled nozzle, and the camera system, as shown in Fig. (6).

The barometric control system consists of the air pump, the 3-way regulating valve of high precision, and gas circuit, and it mainly provide the jetting system with feed pressure and driving pressure of the nozzle. The driving air pressure is a pulse air pressure with the adjusting scope as $0 \sim 1 \mathrm{Mpa}$ and the precision as $0.02 \mathrm{Mpa}$. After the driving pulse generation controller has received the controlling parameters, controlling the on-off time of the radiotube would generate pulse air pressure, and changing the electric pulse width of the supplying electromagnetic valve will produce the barometric pulse with needed width. The static feed pressure with the precision of $0.005 \mathrm{Mpa}$ may be obtained through regulating the 3-way regulating valve with high precision. 


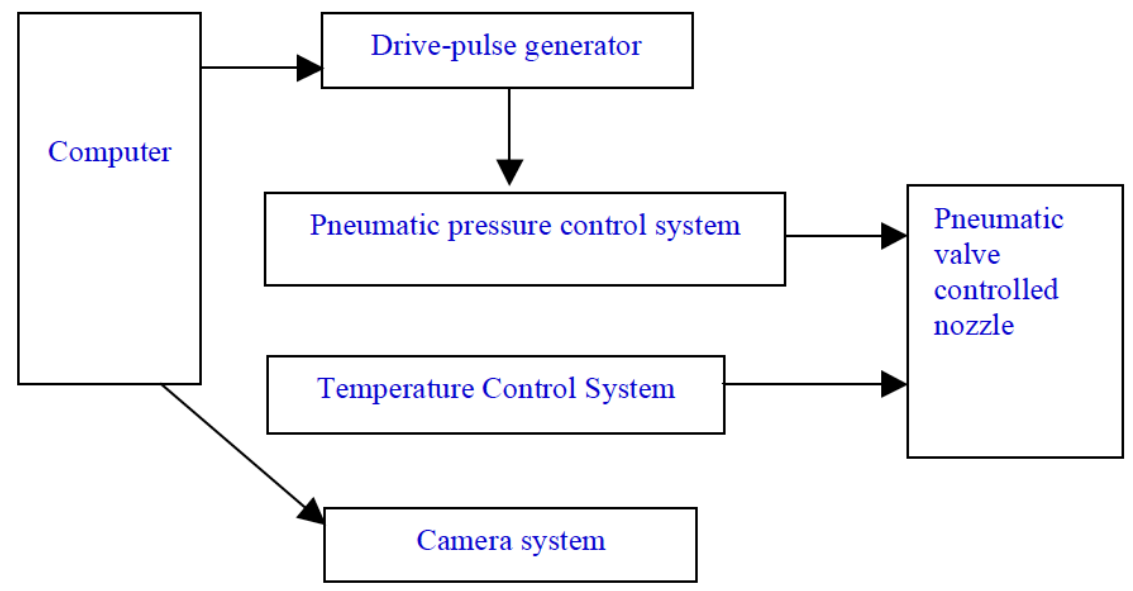

Fig. (6). The experimental platform.

Table 1. Major performance parameter of the platform.

\begin{tabular}{|c|c|c|c|c|c|}
\hline Parameter & Feed Pressure/ Mpa & Driving Pressure/ Mpa & Pulse Width/ $\boldsymbol{\mu s}$ & Striker Stroke/mm & Delay time/ $\boldsymbol{\mu s}$ \\
\hline \hline Adjusting scope & $0 \sim 1$ & $0 \sim 0.2$ & -- & $0 \sim 10$ & -- \\
\hline Precision & 0.02 & 0.005 & 1 & 0.1 & 1 \\
\hline
\end{tabular}

The viscosity of the working liquid has significant effect on the jetting process, and because the viscosity of most of the liquid could be greatly affected by temperatures, so it is of great significance to change obtain changed viscosity parameter through changing the temperature of the working liquid or to make the temperature to be constant so as to raise the precision of the jetting and the accuracy of the experiment. The temperature controlling system has the capability of heating and maintaining constant temperature so as to realize the precise controlling with the error as $\pm 0.1^{\circ} \mathrm{C}$ when the temperature reaches the scope above $80^{\circ} \mathrm{C}$.

The camera has been equipped with a zooming lens of magnification as high as about 20 100 times for observing the process of the formation of droplets during the jetting process.

The pneumatic valve controlled nozzles have been equipped with various kinds of aperture specifications with the depth of the hole as $0.1 \mathrm{~mm}$ and the diameter of the pneumatic piston as $15 \mathrm{~mm}$.

\subsection{Methods for the Strobe Imaging of Droplet Jetting}

In order to reduce the degree of image fuzziness when photographing the targets moving at high speed, the exposure time must be reduced so as to render the amount of movement of the photosensitive element under the status of exposure is comparatively small and even nearly still.

When illuminating time of the light source has been controlled within a very short period, the instant status of the droplet moving at high speed will be obtained so as to indirectly realize the precise capturing of every moving status of the droplet moving at high speed. This kind of method realizing the collection of images from droplets moving at high speeds is named as stroboscopic imaging technique.
The stroboscopic imaging method has no too high requirements on the photographing speed of the camera itself. In this research a common CCD vidicon with the frame rate as $120 \mathrm{fps}$ has been used and the LED illuminant has been taken as the light for the photographing. The LED element is of rather high working frequency, and it is very easy to realize flicker from small to us grade in addition that within such a short illuminating period, the moving target may be regarded as almost motionless. Identically, because the illuminating time of the light source is extremely short, so it is usually unable to obtain enough light pressure to achieve an image during the process of a strobe. Therefore, many times of strobes should be conducted on the same point of the movement of the droplets within a single time of collection of CCD so that CCD may be able to acquire enough light sources so as to collect clear images.

According to the aforementioned analysis it could be known that the stroboscopic imaging requires the application scenarios to have periodically repeating observing targets, the changing of the repetitive period to be sufficiently slow, and the droplet jetting system to be identical with the jetting process under the same driving signal, only in which way could every time of strobe could obtain identical image from the same position so as to ensure the correct overlapped imaging.

When the droplet jetting is observed, the frequency of the droplet continuous jetting is identical to the strobe frequency so that every droplet will have image in the CCD during strobe. When the exposure time of a frame of CCD has been exhausted, what will be obtained is actually a piece of photo where several consecutive droplets are overlapped at one point. If the phase relation between the jetting pulse and strobe pulse is changed, then the position on which the droplet is shown on the picture will also be changed. 


\section{EXPERIMENTAL METHODS AND RESULTS ANALYSIS}

\subsection{The Fundamental Relationship Between the Viscosity Differences and the Droplet Jetting Process}

The striker stroke was set as $0.5 \mathrm{~mm}$, the jetting temperature was at a constant $50^{\circ} \mathrm{C}$, the driving barometric pressure was $0.5 \mathrm{Mpa}$, the feed pressure is $0.02 \mathrm{Mpa}$, the driving pulse width is $10 \mathrm{~ms}$. Water immiscible glycerol was taken to obtain two kinds of working liquids, among which the viscosity of Liquid A was $200 \mathrm{mPa} \cdot \mathrm{s}$ at the temperature of $25^{\circ} \mathrm{C}$ while that of Liquid B was $260 \mathrm{mPa} \cdot \mathrm{s}$. Fig. (7). has shown the process of the jetting and generation of the two kinds of working liquid droplets that were different from each other in viscosity through comparison. In the figure, the jetting system is in a non-working status at the time point of (a) and the edge of the nozzle is a clear straight line. (b) (j) had shown the complete process of the jetting out-stretchingparting-contracting into droplets of the liquid.

It could be clearly seen from (b), (c), and (d) that the liquid pushed out through knocking is continuously stretched out in the shape of a column, the local pressure at the nozzle had been reduced, and the jetting speed at the end of the liquid column has been reduced to be lower than the speed at the front end. It could be seen that the liquid column had begun to break into two sections from (e) (j), and the latter section had a too small speed to overcome the surface tension and viscosity resistance, so it was just piled adjacent to the surface of the nozzle again, while the former section continued to fly forward. The reason for the breaking of the liquid column lies in the tightening effect of the jet fluid. At first the liquid at the former part had a tail, but finally it was integrated into a ball under the effect of the surface tension, such as $(\mathrm{j})$.

The conditions set by the experiment are ideal to both the two kinds of liquids. It could be seen from the results of the various measurement that the viscosity has significant effect on the process of the droplet jetting; the amount jetted by Liquid B was smaller than that of Liquid A, while at the same moment the jetting distance is also rather small, and the liquid column had tended to break at $300 \mathrm{us}$, which was earlier than Liquid A. Therefore, in order to obtain rather small droplet jetting, the viscosity of the working liquid had been raised, for example, it was practicable to reduce the temperature of the jetting. In the meanwhile, because of the sensitivity of the viscosity of the most liquids to temperature, the working temperature must be constant so as to obtain stable jetting effect.

\subsection{Striker Stroke, Liquid Viscosity and the Shape of the Droplets}

In order to further study the process of the striker affecting the diameter of the droplets, the experiment uses the glycerin solution with the viscosity as $200 \mathrm{mPa} \cdot \mathrm{s}$ at $25^{\circ} \mathrm{C}$ as the jetting material with the jetting temperature as $70^{\circ} \mathrm{C}$, the driving pressure value as $1 \mathrm{Mpa}$, the feed material pressure as $0.1 \mathrm{Mpa}$, and the driving pulse width as $100 \mathrm{~ms}$. This group of parameter may be sufficient to ensure that the striker in every jetting could be raised to the limit of the stroke. The strokes of the regulating strikers were $0.5,0.6$,
$0.9,1.2,1,5,1.8$, and $2.0 \mathrm{~mm}$. The relationship between the diameters of the droplets and the striker stroke has been recorded, as shown by Fig. (8). It has been shown by the experimental results that within a certain scope the increasing stroke of the striker would also gradually increase the diameter of the droplet, and this result conforms to common predictions.

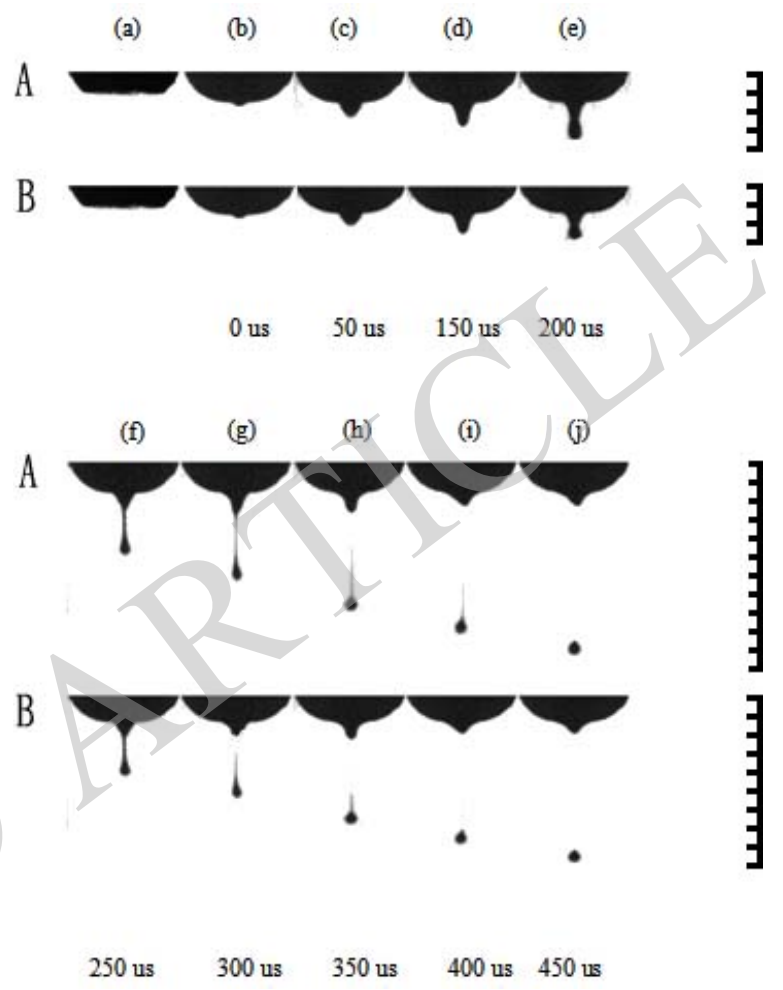

Fig. (7). The process of the formation of the droplets with different viscosities.

It has been found in the experiment that when the striker stroke was at $0.5,0.6$, or 0.9 , the jetting shapes of the droplets were all very good. When the striker stroke was too large, the droplets at the nozzle would not form the spherical head, which was shown as irregular splashing jetting. The reason is considered as that at the time when the too large stroke was knocking the nozzle the energy obtained by the working liquid was much more than the surface tension and viscous resistance of the fluid itself so as to form disordered jetting, see (a) in Fig. (9).

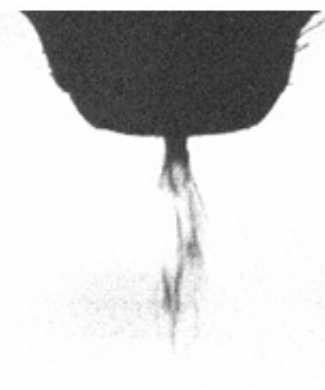

(a)

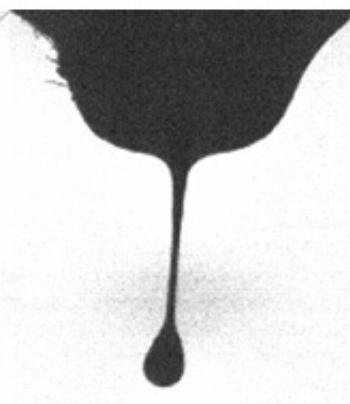

(b)
Fig. (8). Disordered jetting. 
The viscosity of the working liquid was changed to be $400 \mathrm{mPa} \cdot \mathrm{s}\left(25^{\circ} \mathrm{C}\right)$ with other conditions unchanged for repeating the experiment, and then it was found that the minimum striker stroke needed to be raise to be above $0.9 \mathrm{~mm}$ so as to ensure the stable jetting of the droplet in addition that no disordered jetting was found even when the stroke used was $1.8 \mathrm{~mm}$. It was obvious that when the striker stroke was too small and the local pressure thus incurred was too small, then the energy obtained by the droplet was rather small and it is not sufficient for overcoming the surface tension and viscosity resistance, thus no jetting would be allowed.

It could be known from the comparison of the jetting process of these two kinds of liquids that the slightly higher viscosity could reduce the difficulty in adjusting the pneumatic valve controlled nozzle and thus allow the striker stroke to have larger stroke scope.

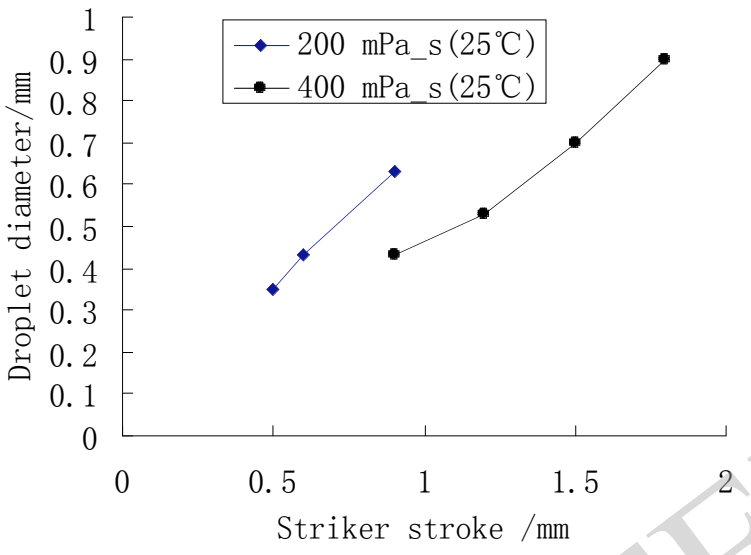

Fig. (9). The striker stroke and droplet diameter under two kinds of viscosities.

\subsection{Several Kinds of Tiny Droplet Jetting Phenomena with Bad Stability}

To produce tiny droplets with smaller diameters through jetting liquids of high viscosity is of important value in engineering application. In the experiment several possible schemes were found. The experiment had used glycerin solution with the viscosity as $400 \mathrm{mPa} \cdot \mathrm{s}$ at $25^{\circ} \mathrm{C}$ was taken as the jetting material in the experiment, the jetting temperature was $70^{\circ} \mathrm{C}$, the feed pressure was $0.03 \mathrm{Mpa}$, the striker stroke was $1.6 \mathrm{~mm}$, the driving pulse width was $20 \mathrm{~ms}$, the driving barometric pressure was $0.5,0.6,0.7,0.8$, or 0.9 Mpa respectively. Fig. (10). has shown the curve of the relationship between the measured diameter of the droplets and the driving barometric pressure.

When the driving barometric pressure was reduced to be below $0.8 \mathrm{Mpa}$, the diameter of the droplets began to reduce dramatically, and when it was $0.5 \mathrm{Mpa}$ even the independent liquid droplets with the diameter below $0.1 \mathrm{~mm}$ and in good shape could be seen again, the reason for which lies in that within the $20 \mathrm{~ms}$ of driving pulse width time smaller barometer pressure has been unable to raise the striker to the limit of the stroke, thus less working liquid could enter the nozzle and the impact force of the striker was also small, and both the reasons had resulted in the contracting of the diameters of the droplets.
The aforementioned parameters were maintained unchanged and only the nozzle was changed or readjusted, after which the experiment was repeated for several times and the thus obtained values of the diameters of the minimum liquid droplets were of bad repetitiveness. The conclusion was that under the current experimental conditions and levels of nozzle technology, the striker was in a status of incomplete lifting and the does not have sufficient stability, so it is not suitable for it to be applied to engineering of tiny droplet jetting.

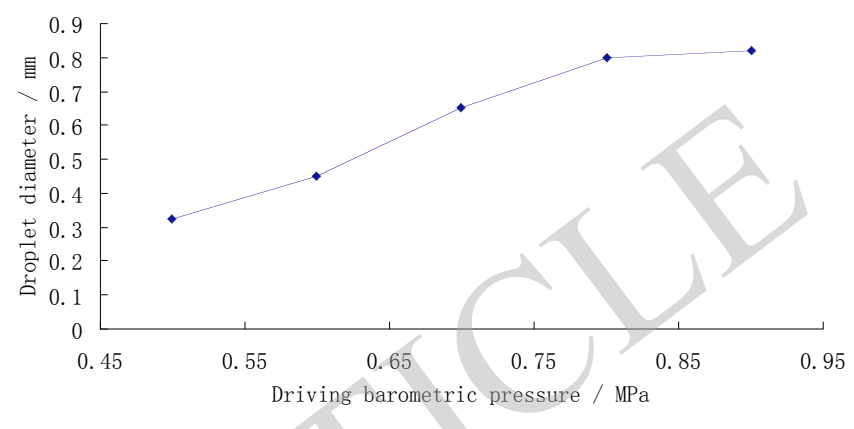

Fig. (10). The curve of the relationship between the driving barometric pressure and the droplet diameter.

Compared to the effect of the reducing of the driving pressure, reducing the driving pulse width may lead to the incomplete lifting of the striker. The aforementioned parameter may still be used, and the lengths of the controlling driving width were $5,10,15,20$, and $25 \mathrm{~ms}$. Fig. (11). showed the result of the jetting. When the driving pulse width was too small, the droplets had the phenomenon of on-and-off phenomenon and became unstable, which was similar to the effect of the driving barometer pressure value on the diameter generated by the droplet.

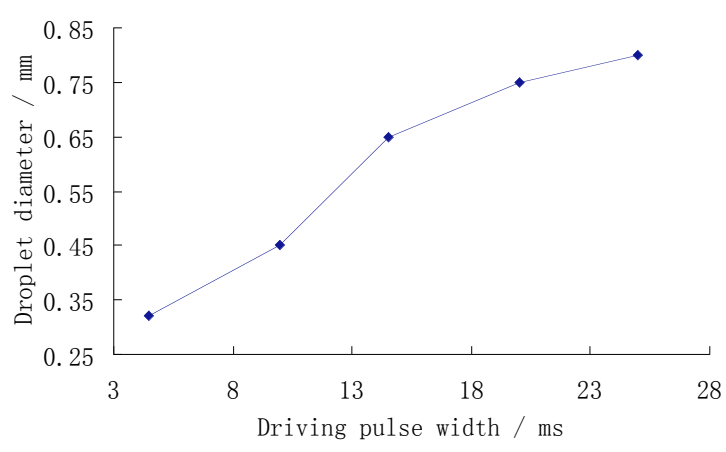

Fig. (11). The curve of the relationship between the driving pulse width and the droplet diameter.

\subsection{The Liquid Residue on the Nozzle Affected the Jet- ting}

It had been found in the experiment that the feed pressure could directly affect the micro droplets. Glycerin solution was used in this experiment as the jetting material with the viscosity as $400 \mathrm{mPa} \cdot \mathrm{s}$ at $25^{\circ} \mathrm{C}$, the jetting temperature is $70^{\circ} \mathrm{C}$, the striker stroke is $0.6 \mathrm{~mm}$, the driving barometer pressure value was $0.6 \mathrm{Mpa}$, the driving pulse width was $40 \mathrm{~ms}$, and the curve of the relationship between the diameter and feed pressure was shown in Fig. (12). 


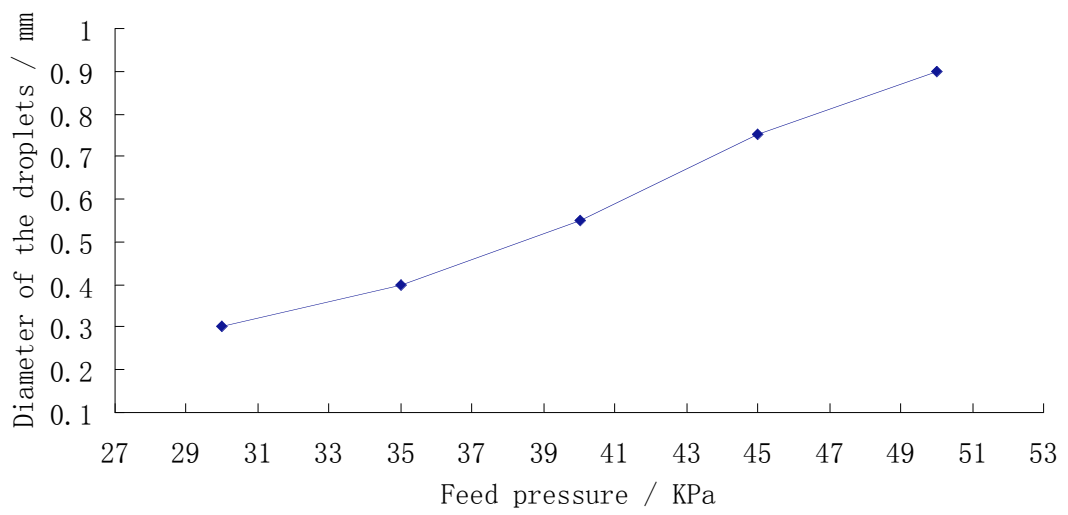

Fig. (12). The curve of the feed pressure and the diameter of the droplets.

It has been shown by the results of the experiment that the diameter of the droplets within a certain scope was increased with the increasing of the feed pressure, which was consistent to the theoretical analysis. It has also been found within the experiment that when other parameters were fixed and the pressure of the materials supplied exceeded a certain value, the liquid would gradually get piled on the surface of the nozzle and cover the whole surface of the nozzle due to the excessive feed pressure and the nozzle could not jet droplets in a normal way, as shown by Fig. (13).

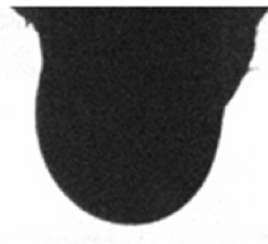

(a)

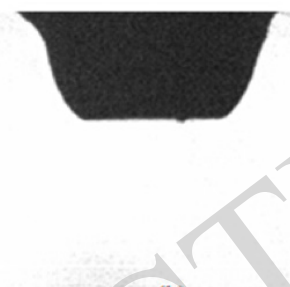

(b)

\section{CONCLUSION}

The results of the observation of this research were identical to the primary theoretical analysis. The stroke imaging method is the effective measure for the observation of pneumatic valve controlled droplets jetting.

The factors like striker stroke, the pulse pressure, and feed pressure would all affect the shape of the liquid droplets. All these parameters were of positive correlation to the diameter of the droplets. Controlling the striker stroke is the most effective and stable method for changing the diameter and shapes of the droplets, and as long as large feed press and pulse barometric pressure may be provided and the width of the driving pulse is wide enough, the diameter of the liquid droplets may be related to the striker stroke within a larger scope.

When the pulse barometric press or the driving pulse width is small and the striker has not been completely raised to the extreme position during work, the nozzle may jet out very tiny droplets under this circumstance. But the stability of this kind of status is rather bad, it is hard for the shape of tiny liquid droplets to be shown again after readjusting the nozzle. Therefore, it is not suitable for the circumstances in need of stable and consistent liquid droplets.

Sufficient feed pressure is advantageous for stable jetting, but the excessive feed pressure may render the volume of the latter section after the breaking of the liquid column

\section{CONFLICT OF INTEREST}

The authors confirm that this article content has no conflict of interest.

\section{ACKNOWLEDGEMENTS}

This work is supported by the Innovation team fund of Zhejiang Province, China (No.2011R50011).

\section{REFERENCES}

[1] C. Gao, S. Huang, and L. Chen, "Progress of the Application of Droplet Jetting Technology," Journal of Inorganic Chemical Materials, vol. 19, no.4, pp. 719-722, 2004.

[3] L. Li, L. Qi, and F. Yang, "Numerical Simulation of Flow Field and Temperature Field during the Jetting Process," Acta Aeronautica et Astronautica Sinica, vol. 28, no. 3, pp. 719-722, 2007.

Fig. (14). The phenomenon of jetting deviation. 
[4] L. Jiang, Z. Jia, and D. Guo, "Status of the Researches on the Researches of Jetting and Printing Forming Technology," Meeting of Rapid Prototyping and Rapid Manufacturing, pp. 21-26, 2006.

[5] D. Wei, R. Zhang, and R. Wu, "The Mathematical Model of the Process of the Piezoelectric Driving Droplet Jetting," China Mechanical Engineering, vol. 16, no. 7, pp. 611-614, 2005.

[6] T. Mu, "Forced Vibration of the Two-layered Thin Round Table Constituted by Metal and piezoelectric ceramic," Acta Acustica, no. 9, pp. 298-303, 1984.

[7] D. Wei, R. Zhang, and R. Wu, "Designing of the Piezoelectric Droplet Spray Device," Journal of Tsinghua University (Science and Technology), vol. 44, no. 8, pp. 1107-1110, 2004.

[8] K. K. B. Hon, L. Li, and I. M. Hutchings, "Direct writing technology-advances and developments," CIRP Annals-Manufacturing Technology, vol. 57, no. 2, pp. 601-620, 2008.

[9] J. Burgold, F. Weise, M. Fischer, G. Schlingloff, T. Henkel, J. Albert, G. Mayer, and A. Schober, "Evolution and operating experiences with different drop-on-demand systems," Macromolecular Rapid Communications, vol. 26, no. 4, pp. 265-280, 2005.

[10] H. Huang, L. Qi, F. Yang, and X. Jiang, "Uniform droplets forming technology and application," Manufacturing Technology \& Machine Tool, vol. 6, pp. 1-9, 2008.

[11] C. Gao, S. X. Huang, L. Chen, C.H. Liu, X.N. Liu, and J. Bao, "Progress in inkJet technique and its applications," Journal of Inorganic Materials, vol. 4, pp. 1-18, 2004.
[12] D. Dixon, J. Kazalski, and F. Murch, "Practical issues concerning dispensing pump technologies-adhesive bonds to a surface in a way that is directly proportional to its area," Circuits Assembly, vol. 8, no. 8 , pp. $36-41,1997$.

[13] C. Q. Ness, and A. R. Lewis, "Step-by-step: pathways to smt part 5-adhesives/epoxies \& dispensing," Surface Mount TechnologyLibertyville, vol. 12, no. 5, pp. 114-123, 1998.

[14] F. Liu, R. Wu, and R. Zhang, "Application of the Jetting Technology of in Bio Manufacturing Engineering," Chinese Journal of Mechanical Engineering, vol. 42, no. 12, pp. 13-20, 2007.

[15] S. Zhou, and J. Xi, "Experimental Researches on the Simulation and Scale Uniformity of the Piezoelectric Driving Diaphragm Typed Droplet Jetting," Chinese Journal of Mechanical Engineering, vol. 49, no. 8, pp. 178-185, 2013.

[16] H. K. Suh, S. W. Park, and C. S. Lee, "Effect of piezo-driven injection system on the macroscopic and microscopic atomization characteristics of diesel fuel spray," Fuel, vol. 86, no. 17, pp. 28332845, 2007.

[17] P. Ben-Tzvi, R. B. Mrad, and A. A. Goldenberg, "A conceptual design and FE analysis of a piezoceramic actuated dispensing system for microdrops generation in microarray applications," Mechatronics, vol. 17, no. 1, pp. 1-13, 2007.

Received: May 26, 2015

Revised: July 14, 2015

Accepted: August 10,2015

(C) Zhan et al.; Licensee Bentham Open.

This is an open access article licensed under the terms of the (https://creativecommons.org/licenses/by/4.0/legalcode), which permits unrestricted, noncommercial use, distribution and reproduction in any medium, provided the work is properly cited. 